\title{
1 Early mechanisms of whisker development: Prdm1 and its regulation in whisker development 2 and evolutionary loss
}

3

4

Pierluigi Giuseppe Manti ${ }^{1,2,3, *}$, Fabrice Darbellay, ${ }^{4,5}$, Marion Leleu ${ }^{6}$, Bernard Moret ${ }^{1}$, Julien Cuennet ${ }^{1}$, Frederic Droux ${ }^{1}$, Magali Stoudmann ${ }^{1}$, Gian-Filippo Mancini ${ }^{7}$, Agnès Hautier ${ }^{7}$ and Yann Barrandon ${ }^{1,8,9,10,11^{*}}$

1: Laboratory of Stem Cell Dynamics, School of Life Sciences, Ecole Polytechnique Fédérale Lausanne, 1015, Lausanne, Switzerland.

2: Department of Oncology ed Hemato-Oncology, Università degli Studi di Milano, Milan, Italy. Pierluigi.manti@unimi.it

3: Department of Experimental Oncology, IEO, European Institute of Oncology, IRCCS, Milan, Italy. Pierluigi.manti@ieo.it

4: Laboratory of Developmental Genomics, School of Life Sciences, Ecole Polytechnique Fédérale Lausanne, 1015, Lausanne, Switzerland.

5: Environmental Genomics and Systems Biology Division, Lawrence Berkeley Laboratories, Berkeley, United States

6: Bioinformatics Competence Center, Ecole Polytechnique Fédérale Lausanne, 1015, Lausanne, Switzerland.

7: Histology Core Facility, Ecole Polytechnique Fédérale Lausanne, 1015, Lausanne, Switzerland.

8: Centre Hospitalier Universitaire Vaudois, 1011, Lausanne, Switzerland. yann.barrandon@epfl.ch.

9: Duke-NUS Graduate Medical School, Singapore, Singapore. yann.barrandon@epfl.ch. 10: Department of Plastic, Reconstructive and Aesthetic Surgery, Singapore General Hospital, Singapore, Singapore. yann.barrandon@epfl.ch.

11: Skin Research Institute of Singapore A*STAR, Singapore, Singapore. yann.barrandon@epfl.ch. *: to whom correspondence should be addressed 


\begin{abstract}
28 Abstract
29

30 Whiskers (vibrissae) are miniaturized organs that are designed for tactile sensing. Extremely 31 conserved among mammals, they underwent a reduction in primates and disappeared in the 32 human lineage. Furthermore, whiskers are highly innervated and their mechanoceptors signal to 33 the primary somatosensory cortex, where a column of neurons called "barrel" represents each of 34 them. This structure, known as barrel cortex, occupies a large portion of the somatosensory cortex 35 of the rodent brain. Strikingly, $\operatorname{Prdm1}$ conditional knockout mice are one of the rare transgenic 36 strains that do not develop whisker hair follicles while still displaying a pelage (Robertson et al. 37 2007). Here we show that Prdm1 is expressed early on during whisker development, more 38 precisely in clusters of mesenchymal cells before placode formation. Its conditional knockout leads 39 to the loss of expression of Bmp2, Shh, Bmp4, Krt17, Edar, Gli1 though leaving the $\beta$-catenin driven 40 first dermal signal intact. Furthermore, we prove that Prdm1 expressing cells not only act as a 41 signaling center but also as a multipotent progenitor population contributing to the formation of 42 the dermal papilla, dermal sheath and pericytes of the vascular sinuses of vibrissae. We confirm by 43 genetic ablation experiments that the absence of motile vibrissae (macro vibrissae) formation 44 reverberates on the organization of nerve wiring in the mystacial pads and organization of the 45 barrel cortex. We prove that Lef1 acts upstream of Prdm1 and identify a potential enhancer 46 (named Leaf) that might be involved in the evolutionary process that led to the progressive 47 reduction of snout size and vibrissae in primates.
\end{abstract}




\section{Introduction}

Whiskers (or vibrissae - from the latin vibrio) are exquisite miniaturized sensory organs specialized in tactile sensing (Petersen 2007), (Brecht 2007), (Diamond et al. 2008). Conserved throughout evolution, whiskers underwent a reduction throughout the primate adaptive radiation (Van Horn 1970) and disappeared completely in the human lineage; yet vestiges of whisker capsular skeletal muscles remain in the human upper lip (Tamatsu et al. 2007). Those specialized hair follicles are bigger both in length and width compared to pelage ones and are enveloped by vascular sinuses conferring rigidity to the hair shaft. Fibers of striated muscle have an insertion on the capsula and encompass the vascular sinuses. While macro vibrissae are motile and used for distance detecting/object locating, micro vibrissae are immotile and used for object identification.

The processing of whisker-acquired information occurs in the barrel cortex, where each whisker is represented by a discrete and well-defined cytoarchitectonic structure that goes under the name of barrel (Woolsey and Van der Loos 1970). The barrel map occupies a large area of the brain, it is in large part genetically specified and forms early on during development (Erzurumlu and Gaspar 2012). As whisker pattern is established earlier and independently from innervation, the hypothesis being that whiskers impose their own pattern onto the somatosensory cortex in the homeomorphic fashion has arisen (Andrés and Van der Loos 1985).

Prdm1 is a zinc-finger transcriptional repressor (Keller and Maniatis 1991) that has been proven to be a master regulator controlling terminal differentiation of B-lymphocytes (Angelin-Duclos et al. 2000) (Shaffer et al. 2002) (Shapiro-Shelef et al. 2003); it also governs T-cell homeostasis (Martins et al. 2006) and primordial germ cells specification (Vincent et al. 2005), stem cell maintenance in the sebaceous gland (Ohinata et al. 2005) and skin differentiation (Horsley et al. 2006). Prdm1 has also been shown to play a crucial role during whisker development (Robertson et al. 2007).

Prdm1 (also known as Blimp1) conditional knockout mice are one of the very rare transgenic animals entirely lacking whisker (vibrissae) follicles while pelage hair follicles develop physiologically. The loss of this gene impairs whisker development (Magnúsdóttir et al. 2007), though the exact stage at which the development is halted has not been identified yet. Furthermore, it is still not known which type of mystacial vibrissae (macro- and/or micro-) are impacted by Prdm1 knockout.

Moreover, Robertson et al. proved that Prdm1 positive mesenchymal cells give rise to the mature dermal papilla and expand to form a mesenchymal layer immediately surrounding the hair follicles. However, what this mesenchymal layer gives rise to the adult whisker is a question that has not been answered yet.

Intriguingly, the reverberation of whisker development halt onto the barrel cortex has not been investigated yet. The re-organization of the somatosensory cortex is a phenomenon of great importance for evolutionary reasons, given the expansion of the brain areas dedicated to the processing of the sensory organs that evolutionary took over.

Eventually, little is known on the regulation of Prdm1 during whisker development. Investigating this subject is of fundamental importance as the loss of potential regulatory elements in upstream 
bioRxiv preprint doi: https://doi.org/10.1101/2021.03.11.433122; this version posted March 12, 2021. The copyright holder for this preprint (which was not certified by peer review) is the author/funder. All rights reserved. No reuse allowed without permission.

91 genes might explain the reduction that occurred throughout the primate adaptive radiation and 92 that led to reduction of snout size and vibrissae while hands and eyes of diurnal monkeys took 93 over as sensory organs. 
94

95

96

97

98

99

100

101

102

103

104

105

106

107

108

109

110

111

112

113

114

115

116

117

118

119

120

121

122

123

124

125

126

127

128

129

130

131

132

133

134

135

136

\section{Results}

\section{Prdm1 is a master gene of whisker follicle development}

We localized Prdm1 expression by immunofluorescence in Prdm1 mEGFP embryonic whisker pads during the first stages of whisker development (Figure 1A); those mice dispose of an mEGFP reporter recombined in frame with the exon 3 of Prdm1 (Ohinata et al. 2005) (SFig 1A, Figure 1B). mEGFP expression in heterozygous embryos can be detected in a specific cluster of mesenchymal cells underlying the monolayer of embryonic epidermis (referred as stage 0 of whisker development, Hardy 1992) that will later on form the whisker epidermal placode. It continues to be expressed in this compartment until stage 4, when its expression is turned on in the inner root sheath (IRS) of the follicle. At stage 5, Prdm1 expression disappears in the dermal papilla (Figure 1C). Those results are validated by Prdm1 immunohistochemistry (IHC) in wild type whisker pads (Figure 1D).

On the other hand, Prdm1 expression in the dermal condensate of both head and back pelage hair follicles starts at embryonic day 14.5 (stage 0 of pelage follicle development) but is transient as it disappears at stage 2 (SFig 2A).

To position Prdm1 in the molecular cascade leading to whisker formation, we generated Prdm1 knockout embryos. It has been previously shown that the constitutional knockout of Prdm1 leads to severe impairment of the placenta resulting in early embryonic lethality. Therefore, we used a Sox2Cre deleter strain to bypass placental developmental halt (Vincent et al. 2005, see SFig1B). This transgenic line induces recombination in all epiblast cells by embryonic day (E) 6.5 but little or no activity in other extraembryonic cell types at this time (Hayashi et al. 2002). We were thus able to generate and harvest Prdm1 homozygous conditional knockout embryos (cKO1) until E17, where exons four to eight are deleted by site-specific Cre-mediated recombination (SFig 1C).

We started by analyzing E12.5 and E13 cKO1 embryos, time-points when whisker follicle formation begins. Macroscopically, the cKO1 embryos are smaller compared to their wild type matches; as expected, they lack two or three digits (incomplete adactylia) in the forelimbs (SFig 2B). Microscopically, no whisker placode can be detected, and the dermal condensate formation cannot be observed (SFig 4). On the other hand, the development of pelage hair follicles is not impaired.

To understand where Prdm1 stands in the molecular cascade leading to whisker formation (Figure 2A) we looked with fluorescent in situ hybridization (FISH) at the expression of the genes involved in the first molecular steps of hair follicle morphogenesis both in the epithelial and mesenchymal compartment. $\beta$-catenin is implied in establishing the first dermal signal (Noramly et al. 1999); consistent with this, Lef1 is expressed in the mesenchyme of the mouse vibrissa pad prior to vibrissa follicle development, and initiation of vibrissa follicle development is dependent on its expression (van Genderen et al. 1994), (Kratochwil et al. 1996). In E13 homozygous cKO1 mice, Lef1 is expressed homogeneously in the mesenchyme of the whisker pad, thus indicating that the first signal is intact; however, the physiological restriction of expression of Lef1 in the whisker placode and underlying mesenchyme does not occur (Figure 2B). 
137 The gene cascade that is activated in placode formation is not activated in Prdm1 cKO1 mice. No 138 placode formation can be observed both macroscopically and microscopically. The patterned 139 upregulation of molecules involved in the promotion and inhibition of placodal fate including $140 \mathrm{Bmp4}$ (Figure 2C) in the pre-follicle mesenchyme and Bmp2 (Figure 2D) in the epithelial compartment does not occur. The same applies for a molecule involved in the first epidermal signal, SHH - secreted by the placodal cells (Figure 2E). Gli1 is not upregulated in the pre-follicular mesenchyme (Figure 2F). We found that Wnt10b is diffusely expressed in the monolayer of epidermal cells in the sites of whisker formation in CKO1 and does not show a marked upregulation in placodes (as in wild type whisker pads) (Figure 2G). Furthermore, RT-qPCR on whisker pads shows a statistically significant decrease of Edar, involved in the promotion of placode, and Keratin 17, whose expression arises within the single-layered, undifferentiated ectoderm of embryonic day 10.5 mouse fetuses giving rise, in the ensuing 48 hours, to the epidermal placodes (McGowan and Coulombe 1998) (SFig 4). Intriguingly, Lef1 is expressed uniformly in the mesenchyme of cKO1 embryonic whisker pads, and the typical restriction to the whisker placode and pre-follicle mesenchyme does not occur.

152

\section{Whisker inducing mesenchyme contributes to the formation of both the dermal sheath and capsula of adult mystacial whisker follicles}

To investigate the proliferative activity of Prdm1 expressing cells, we administered a short pulse (2 hours) of nucleotide analog ethynyldeoxyuridine (EdU) just prior to analysis to Prdm1MEGFP pregnant mice carrying E12.5 embryos. We could observe that Prdm1 expressing cells can be classified into two subpopulations: the quiescent ones - contiguous to the embryonic epithelium and the proliferative ones at the periphery (Figure 3A). We then aimed at identifying the progeny of Prdm1 expressing cells in the whisker follicle by means of lineage tracing. By crossing the Prdm1Cre transgenic strain with ROSAYFP mice (SFig 1D, E), we were able to follow the progeny of Prdm1 expressing cells at different time points during embryonic development (SFig 5). At E12.5, YFP expressing cells are located in the whisker mesenchymal condensate recapitulating the GFP expression in the Prdm1MEGFP embryonic whisker pad. However, at E13.5 it can be observed that the population of YFP positive cells expands and encompasses the area of mesenchyme surrounding the whisker hair germ. Prdm1 can be detected in this area (except for the precursors of the dermal papilla) in whisker pad sections of both Prdm1MEGFP and WT mice at E12.5 and E13.5 (Figure 3B).

Because of Prdm1 expression in both the endothelium and somites, the lineage tracing experiment could not be performed at a time point later than E13.5. However, we observed that Sox 2 is coexpressed with Prdm1 at E12.5 in the whisker mesenchymal condensate (Figure 3B). Consequently, we crossed Sox2CreERT2 and ROSAYFP mice and injected tamoxifen in pregnant females at E12.5 (SFig 1E, F); double transgenic embryos were analyzed at E14.5, E17.5 and P1-3. 48 hours after tamoxifen injection, YFP positive cells were detected in the dermal condensate of the hair germs. As the epidermal down growth proceeds, the mesenchymal YFP cells progressively encapsulate it (Figure 3C). When the whisker follicle reaches its final anatomical configuration, YFP 
180 is expressed by the whole dermal papilla (DP), dermal sheath (DS) and abundantly by cells residing 181 into the vascular sinuses. More precisely, the latter are enmeshed with CD31 positive cells and 182 display a perithelial position: both the latter and the expression of markers such as $\mathrm{Ng2}$, PdgfrB, Tnap (Tissue nonspecific alkaline phosphatase) indicate that they are pericytes (Figure 3D).

\section{Prdm1 genetic ablation leads to the disorganization of the rodent barrel cortex}

To study the impact of whisker loss on the nervous system, we have generated cKO1 embryos and looked at their innervation in the developing whisker pad. In the wild type whisker pad, the afferent branches of the infraorbital nerve (ION) encapsulate the whisker mesenchymal condensate without penetrating it; on the contrary, they terminate as free nerve endings in the $\mathrm{KO}$ counterpart (Figure 4A).

To evaluate the consequences of the impaired whisker innervation on the nervous system, we have adopted the $W n t 1 C$ re as a deleter strain in order to bypass the neonatal lethality that occurs in cKO1. Wnt1 is expressed virtually in all neural crest derivatives, including the whisker pad mesenchyme and, especially, the mesenchymal compartment surrounding the developing whisker follicle. Wnt1Cre driven Prdm1 conditional KO mice (cKO2 - see SFig 1E) are viable and lack almost all the macro vibrissae except for the 1-3 distal ones of the first row, as observed both macroscopically and microscopically (SFig 6). We retrieved the brains of both WT and cKO2 mice after p21 to account for the developmental maturation of the somatosensory system and sectioned their flattened somatosensory cortex tangentially to visualize the organization of the barrel cortex (Figure 4B). The cytochrome oxidase staining revealed that $\mathrm{CKO} 2$ barrel cortex undergoes a major rearrangement; the residual macro vibrissae are represented by enlarged barrels; the barrels corresponding to the micro vibrissae are, however, still present, even though their pattern is highly disorganized (Figure 4C). To exclude the expression of Prdm1 in the developing barrel cortex - and thus that the barrel cortex phenotype can be ascribable to the loss of Prdm1 in the nervous system - we crossed Prdm1Cre with ROSAYFP mice and looked at YFP expression in the cerebral cortex (Figure 4D). YFP is expressed only by endothelial cells and not by the thalamocortical axons or by layer 4 cortical neurons, thus excluding the aforementioned possibility.

\section{Lef1 acts upstream of Prdm1 in the whisker hair follicle developmental cascade}

We reasoned that $\beta$-catenin/Lef1 might act upstream of $\operatorname{Prdm} 1$ during whisker follicle development. To prove this, we obtained Lef1 constitutional KO embryos by crossing homozygous Lef1 ${ }^{\text {tm1Rug }}$ mice (see SFig1G) (Van Genderen et al 2004) and investigated Prdm1 expression in the E12.5 whisker pad both at the mRNA and protein level.

At a molecular level, the quantity of Prdm1 transcript in the Lef1 KO embryos is lower compared to the wild type counterpart, as demonstrated by RT-qPCR (Figure 5A). We sectioned the E12.5 whisker pads from Lef1 KO and WT mice and localized Prdm1 by IHC, focusing on the mesenchymal cells located under the characteristic surface elevations of the whisker pad that constitute the sites of whisker placode induction - from now on referred as areas of analysis. We analyzed the transverse sections of the embryonic whisker pad through (a) the primitive nasal 
223 cavity (b) the vomeronasal organ and (c) the tongue of five Lef1 KO embryos. Out of five Lef1 KOs,

224 three areas did not express Prdm1. As for the remaining two embryos, we could observe Prdm1 225 expression in 2/28 and 4/27 areas (Figure 5B and 5C).

226

227

228

229

230

231

232

233

234

235

236

237

238

239

240

241

242

243

To understand if the de-regulation of Prdm1 and/or Lef1 might help to explain vibrissae reduction and their eventual loss in humans, we looked at their regulatory regions. As for Lef1, a transposable enhancer trap mapping to a particular locus (chr3:130,927,182-130,927,529 in mm10 assembly) suggests that its regulatory region is located on the centromeric side of the gene, in the adjacent gene desert (TRACER LacZ expression database, SB line name 183038-emb20) (Chen et al. 2013). The transgene expression can be observed at E11.5 in the brain, mammary glands, whisker pad and the tip of tail, tissues/organs where Lef1 is physiologically expressed during development (Figure 6A).

We compared the multispecies alignment of animals with and without whiskers in the aforementioned locus searching for putative regulatory elements. Conservation scored by PhastCons indicated the presence of an element (878bp) conserved throughout the two categories of species (chr3:131,019,746-131,020,624) which contains a sub-region $(521 \mathrm{bp})$ specifically absent in animals deprived of functional whiskers (human, chimp, gorilla, gibbon, rhesus, baboon and squirrel monkey) mapping chr3:131,020,103-131,020,624 (mm10 assembly) (Figure 6B). Altogether, this DNA element (Leaf, chr3: 131,019,746-131,020,624) is located in the same topological associating domain (TAD) where Lef1 resides (Figure $6 C$ ).

To observe if this region can contact the promoter of Lef1, we performed 4C-Seq. The analysis was conducted on whole microdissected whisker pads at E12.5 using primers positioned in the promoter of Lef1. Adult kidney was used as the negative control as Lef1 is not expressed in this tissue (Figure 7A and 7B, SFig 7, Darbellay and Necsulea, 2020).

We found that Lef1 promoter scores contact mainly in cis, within an area of around $700 \mathrm{~kb}$ surrounding the Lef1 locus, corresponding to its TAD. More specifically, the centromeric region contains most of the peaks of interaction, whereas telomeric contacts occur chiefly with the coding sequence of Lef1, extending until the end of the coding sequence of the neighboring gene Hadh.

In the E12.5 whisker pad, the promoter of Lef1 contacts several centromeric regions, among which the one containing the primate-specific deletion (Leaf). We proceeded to quantify the number of contacts that the Lef1 promoter establishes with Leaf in the E12.5 whisker pad and the adult kidney both in the normalized and profile corrected dataset; we represented the sum of the fragments through boxplots. We found high statistical significance between the scores mapping Leaf in the E12.5 whisker pad compared to the adult kidney (****, Mann-Whitney test, $p=0.0008$ in normalized and $\mathrm{p}<0.0001$ in $\mathrm{PC}$ ).

In the reciprocal experiment, we looked at the contacts from the murine Leaf enhancer with the promoter and coding sequence of Lef1. We found high statistical significance between the scores mapping Lef1 in the E12.5 whisker pad compared to the adult kidney, suggesting productive longrange interactions between Leaf and Lef1 in the whisker pad (****, Mann-Whitney test, $p<0.0001$ in normalized and $p<0.0001$ in $\mathrm{PC}$ ), thus confirming what observed in the specular viewpoint. 
266 It has been recently demonstrated that a specific enhancer of the androgen receptor (Ar) is 267 expressed in the whisker mesenchyme during development (McLean, 2011); the authors propose 268 that the loss of this enhancer is associated to the loss of both sensory vibrissae and penile spines in 269 the human lineage. Notably, the castration or genetic deletion of the Ar results in a reduced 270 growth of whisker follicles in mice without their full disappearance.

271 To understand if the AR is involved in the early steps of whisker development together with Prdm1 272 and Lef1, we performed both IHC and RT-qPCR on embryonic whisker pads at E12.5. The AR IHC 273 shows no signal either in the whisker placodes or in the mesenchyme underneath it at E12.5; the 274 RT-qPCR confirmed the absence of expression in the micro-dissected whisker pads at E12.5 and E13.5 (Figure 7C), thus excluding the hypothesis of its early involvement in whisker formation.

\section{Discussion}

Overall, our results prove that (i) Prdm1 acts at the level of the first dermal signal during whisker development and its expression is regulated by $\beta$-catenin/Lef1 (ii) Prdm1 is fundamental for the proper functioning of the whisker signaling center that will later contribute to the formation of the adult structures of the whiskers, more specifically the dermal sheath, the dermal papilla and the pericytes (iii) the disappearance of macro vibrissae induced by the genetic ablation of Prdm 1 causes the reorganization of the entire murine barrel cortex (iv) Lef1 is positioned upstream of Prdm1 in the whisker inductive cascade and the loss a putative regulatory element (Leaf) might contribute to the multi-step process that lead to the progressive de-functionalization and loss of whisker in primates and humans.

Prdm1 has already been described as a key gene for whisker development (Robertson et al. 2007). We prove that Prdm1 is expressed in clusters of mesenchymal cells before whisker placode appearance, thus implying a very early role in whisker formation. Our in-situ hybridizations on the Sox2Cre driven Prdm1 KO confirm and expand the previous results of Robertson et al. We prove that in Prdm1 cKO1 whisker pads the $\beta$-catenin based first dermal signal is intact; however, no placode formation occurs - as shown by Wnt10b, Gli1, Edar, Krt18 ISH - and the first epidermal signal cannot occur. Thus, we can conclude that Prdm1 operates at the level of the first dermal signal.

The fate mapping study conducted by Robertson et al. (Robertson et al. 2007) indicates that Prdm1 expressing cells contribute to the formation of the DP and that the cells failing to be incorporated in the latter migrate to surround the hair shaft. To understand the fate of those specific cells, we performed a lineage tracing study using the Sox2CreERT2 strain, once proven the co-expression of Prdm 1 and Sox 2 in the cluster of mesenchymal cells right underneath the pre-placodal epithelium. Our results clearly illustrate that the population of cells expressing Prdm1/Sox 2 gives rise to several lineages of the adult whisker, thus representing a population of multipotent progenitors. As expected, the mesenchymal pre-condensate contributes to the formation of the dermal papilla; interestingly we demonstrate that it also gives rise to the dermal sheath of the follicle, thus explaining the common inductive properties of the DP and DS (Oliver 1966), Eventually, we prove 
308 that it gives rise to pericytes residing in the whiskers' vascular sinuses; their lineage is confirmed

309 by their anatomical position and the expression of specific markers.

311 Robertson et al. shows that Prdm1 expressing cells display no Ki67 expression and are thus not 312 cycling. Our Edu uptake experiments show that the vast majority of them - the ones that are the 313 most proximal to the placode - do not incorporate Edu, thus confirming Robertson's data. 314 However, we can observe that $\operatorname{Prdm} 1 /$ Sox 2 positive peripheral cells are proliferative. We speculate 315 that peripheral cells progressively lose Prdm1 expression and are thus able to re-enter cell cycle, 316 whereas those underneath the placode retain its expression, functioning as signaling center during 317 development and in the end constituting the dermal papilla of the mature follicle.

We further investigated the impact of Prdm1 knockout on the murine nervous system. In cKO1 whisker pads, the afferent branches of the infraorbital nerve do not organize into plexuses surrounding the mesenchymal condensates but terminate as free nerve endings. Axonal guidance is most probably absent as for the lack of nerve guidance molecules secreted by Prdm1 positive cells. The Wnt1Cre driven Prdm1 KO (cKO2) mice lack almost all macro vibrissae though retaining micro vibrissae. This result suggests that $\operatorname{Prdm} 1$ plays a key role in macro vibrissae development; on the contrary, the formation of micro vibrissae in CKO2 indicates that the signals required to induce their formation do not rely on Prdm1 and still need to be investigated. Those conditional knockout mice have major rearrangements in the barrel cortex concerning also the representation of micro vibrissae with relevant evolutionary consequences.

Given that the first dermal signal is Wnt-based and that Lef1 plays a crucial role in early whisker development, we decided to focus on the relationship between Lef1 and Prdm1. The downregulation of Prdm1 in Lef1 KO whisker pads both at the mRNA and protein level clearly proves that the $\beta$-catenin-based uniform first dermal signal is indispensable to induce Prdm1 expression. In our model, Prdm1 is dependently arising on the first dermal signal insurgence and as stated above, is fundamental for placode formation. We reasoned that the loss of regulatory sequences in Prdm1, Lef1 or both might explain the multistep evolutionary process that led in the first step to whisker de-functionalization and then disappearance. King and Wilson (Jahoda and Oliver 1984) postulated that "regulatory mutations account for the major biological differences between chimp and human"; it has already been elegantly demonstrated how enhancers regulate

345 It has been previously been shown that the spatial and temporal control of Lef1 expression 346 depends on different regions of the Lef1 locus (Liu et al. 2004). A $2.5 \mathrm{~kb}$ segment of the human 347 promoter can drive $\operatorname{LaCZ}$ expression only in the mesenchymal compartment of the developing 348 whisker follicles; furthermore, it contains a specific element responsive to $W n t 3 a$ and $\beta$-catenin 349 (Liu et al. 2004). Furthermore, the congenital agenesis of molar number 3 (am3) in a murine strain 
350 is attributable to a locus mapping the $130.73-131.69 \mathrm{Mb}$ region of chromosome 3 , suggesting that

351 Lef1 is the strongest candidate for am3 (Shimizu et al. 2013).

352

353

We searched the TRACER database to find the regulatory region of Lef1; the transposon-based enhancer trap indicates that the latter resides centromeric to Lef1 and that is active during whisker morphogenesis at E11.5. We identified a deletion in the regulatory region of Lef1 that is specific to several primates (including human, chimp, gorilla, gibbon, rhesus and baboon) flanking a well-conserved region among many mammals. Leaf falls in the regulatory region identified with the TRACER database and in the TAD where Lef1 resides. We thus postulated that this genomic sequence might have a putative enhancer activity in whisker development and, by modulating Lef1 expression in the whisker pad, and indirectly Prdm1), might be involved in whisker disappearance during evolution. The 4C-seq analyses we performed show that Leaf is contacted by the Lef1 promoter more often in the E12.5 whisker pad (embryonic age when whisker formation starts) compared to control tissue, thus suggesting a tissue-specific interaction. Those results are corroborated by the quantitative analysis of the scores mapping Leaf in the E12.5 whisker pad (and Lef1 in the reciprocal experiment) compared to the adult kidney, clearly underlining a highly statistically significant contact between Leaf and Lef1 promoter and vice-versa occurring specifically in the developing whisker pad. The function of this regulatory element is to be further investigated at the functional level.

When investigating AR expression in the whisker placodes at early stages, we could not detect its presence both at the RNA and at the protein level in both placode and underlying mesenchyme. We can thus rule out an early role of the enhancer that McLean and colleagues has described as involved in whisker loss in humans (McLean et al. 2011). Noteworthy is the fact that 2 strains of AR knockout mice generated by two independent groups (Yeh et al. 2002) (De Gendt et al. 2004) do not display whisker loss. Being it expressed in the mesenchymal compartment at later stages of hair follicle development, we hypothesize that it might play a role more in the de-functionalization of whiskers.

While great apes have lost macro vibrissae though retaining the micro vibrissae on lips (a phenotype that recalls our Prdm1 cKO2 mice), cheeks and eyebrows, humans are the only known mammals to have lost both, though vestiges of vibrissal capsular muscles have been identified in the human upper lip (Tamatsu et al. 2007). In the model we envisage, whisker loss is a multistep process that started during the divergence of the species and that relies on the loss of tissuespecific regulatory elements of several genes involved in whisker formation; more specifically, the loss of Leaf might have contributed to the downregulation of Lef1 expression in the snout of primates, thus contributing to whisker loss together with other mechanisms that have to be identified yet. 
388

389

390

391

392

393

394

395

396

397

398

399

400

401

402

403

404

405

406

407

408

409

410

411

412

413

414

415

416

417

418

419

420

421

422

423

424

425

426

427

428

429

430

\section{Figure Legends}

\section{Figure 1}

(A) Schematic representation of the whisker follicle developmental stages (S1-S4). Whisker development starts around embryonic day 12.5 (day 0 of whisker development) when an early dermal condensate appears in the whisker pad below the embryonic epidermis, which will in turn thicken to form a placode in stage 1 (Figure 5). Subsequently, an epidermal down growth (stage 2) and a dermal papilla (stage 3) are formed. A hollow cone (stage 4) develops by hardening of cells belonging to the hair matrix, thus giving rise to the inner root sheath. Image elaborated on (Hardy 1992). (B) Prdm1MEGFP versus wild type (wt) embryo at e12.5. Fluorescent signal is observed in the developing whisker pad, forelimb, hindlimb and somites. Right below full embryo pictures, magnification of the transgenic and wt whisker pad. (C) Prdm1 IHC on developing whisker pad. Left panel, Prdm1 is expressed in the mesenchymal compartment from stage 1 (S1) to stage 3 (S3) of whisker development. Black dashed circles envelop the areas where Prdm1 is expressed. (D) GFP immunofluorescence on Prdm1MEGFP whisker pads. On the top left, Prdm1 can be detected before placode formation (SO). The GFP expression in the dermal fibroblasts at stage 3 (S3) and in the IRS at stage 4 (S4) confirms that the reporter mouse recapitulates the endogenous pattern of expression of Prdm1. The white dashed lines indicate the epidermal-dermal junction in SO-S3 and demarcate the follicle from the surrounding mesenchyme in $\mathrm{S4}$. The green dotted lines indicate the areas of GFP expression in Prdm1MEGFP mice. Scale bar: $50 \mu \mathrm{m}$.

\section{Figure 2}

(A)Expression of early whisker developmental genes in the Prdm1 cKO1 mouse. Molecular mechanisms underlying the early steps of whisker development. The dermis delivers a Bcatenin based homogenous first signal to the overlying epidermis in order to initiate placode formation. The placode in turns both sustains their growth and inhibit the formation of other placodes in the surrounding epidermis. The promotion of the placodal fate is sustained by several molecules including Wnt10b, Bcatenin/Lef1, Fgf10/Fgfr2-IIlb, Eda/Edar, Noggin, Delta-1/Notch1, whereas the inhibition is based upon Bmp2/Bmp4/Bmpr1a and Delta-1/Notch1. Thus, the placode conveys a first epithelial signal, leading to the clustering of the mesenchymal cells underneath into the dermal condensate. This process mainly relays on $\mathrm{Wnt}$ signaling and $\mathrm{Shh}$. Image modified from (Millar et al. 2002). Fluorescent ISH on e12.5 wild type embryos (S1 of whisker development) reveals that, in physiological whisker development, Lef1 expression is initially homogeneous in the mesenchyme and later confined to the epithelial placode and underlying mesenchyme (B); Bmp2 marks the epithelial placode (C); Bmp4 the underlying mesenchyme (D); Shh is expressed by the placode and induces the condensation of the mesenchyme (E); Wnt10b expression is restricted in placodes (F); Gli1 is upregulated in the pre-follicular mesenchyme. In e 12.5 cKO1 embryos, Lef1 expression remains homogenous throughout the mesenchyme; Bmp2, Bmp4, Shh and Gli1 are no longer detectable wile Wnt10b fails to be upregulated in placode areas; whisker follicles cannot thus reach stage 1 of whisker development. The dashed lines indicate the dermo-epidermal junction. Hybridization is marked with arrows (green arrows indicates expression in the placode, yellow ones in the mesenchyme). Scale bar: $50 \mu \mathrm{m}$. 


\section{$431 \quad$ Figure 3}

432 (A) The vast majority of GFP positive cells do not incorporate Edu contrarily to the peripheral ones 433 at E12.5. (B) The Sox2 immunofluorescence on Prdm1MEGFP whisker pads reveals that Sox2 marks 434 a subpopulation of Prdm1 positive cells. Note that Sox2 is also expressed in the putative 435 oligodendrocytes surrounding the nerve endings surrounding the whisker pre-mesenchymal 436 condensate. Cre expression was induced upon tamoxifen injection in E12.5 Sox2CreERT2/ROSAYFP 437 embryos and examined for YFP expression either in the early stages (E13, E14) or at completion of 438 development (E17, P3). (C) Note that YFP is first expressed in a cluster of mesenchymal cells right 439 underneath the hair germ; when the latter becomes the hair peg, the YFP cluster envelops it into a 440 mesenchymal cup. (D) Analysis at later time-points (E17-P3) reveals the extensive contribution of 441 YFP+ cells to several lineages of the whisker follicle including the DP (starred) and the DS 442 (asterisks). Several YFP+ cells in close contact with the endothelial ones (expressing Cd31) can be 443 observed inside the vascular sinuses; those cells express markers of pericytes such as Tnap, Ng2, 444 Pdgfr $\beta$ (arrows indicate areas of co-expression). White dashed lines (A, B, C) indicate epidermal445 dermal junction. Green dotted lines indicate clusters of GFP positive cells (A, B); yellow dotted 446 lines indicate the progeny of Sox2 positive cells (YFP positive, C). Scale bars: $50 \mu \mathrm{m}, 10 \mu \mathrm{m}(\mathrm{Ng} 2)$, $44720 \mu \mathrm{m}$ (Tnap).

\section{Figure 4}

450 (A) Tuj1 immunofluorescence on Prdm1 cKO whisker pad at E13.5 reveals the innervation process 451 in the early stages of whisker development. Note that the nerve fibers encapsulate the 452 mesenchymal condensate in the wild type whereas they act as free nerve endings in the cKO. (B) 453 Cytochrome oxidase staining on the barrel cortex of Wnt1Cre driven Prdm $1 \mathrm{KO}$ mice. Note the 454 absence of the vast majority of barrels representing the macro vibrissae and the rearrangement of 455 the ones representing the micro vibrissae. (C) Cd31 (red) and GFP immunofluorescence on the 456 somatosensory cortex of Prdm1Cre/ROSAYFP mice. Note that the axons and neurons of layer four 457 of the cortex have never expressed Prdm1 and that YFP+ positive cells are of endothelial origin. 458 The red dotted line in A indicates the area of dermal condensate expressing p75NTR. Scale bar: 50 $459 \mu \mathrm{m}$.

\section{Figure 5}

462 Quantification of Prdm1 by RT-qPCR in both wild type and Lef1 KO E12.5 whisker pads (each dot 463 represents a sample) indicates a severe decrease of Prdm1 expression in Lef1 KO mice compared 464 to the WT counterpart (p-value:0.0007***) (B) Prdm1 IHC on WT and Lef1 KO whisker pads 465 (E12.5). Note the absence of expression of Prdm1 in the ectodermal elevation preconfiguring sites 466 of whisker induction in the Lef1 KO embryos (KO 4, 5, 7). (C) Quantification of Prdm1 expression in 467 Lef1 KO whisker pad (D) Schematic representation of the molecular mechanisms leading to 468 whisker hair follicle formation. (i) A first dermal signal is needed to induce placode formation in 469 the overlying epidermal monolayer (ii) The epithelial placode forms relaying on signals that 470 promote its growth and inhibit placode fate in the surrounding epithelial cells (iii) The placode 471 produces the first dermal signal. 


\section{Figure 6}

474 (A) The TRACER database pinpoints that the regulatory region of Lef1 resides centromeric to his 475 promoter and that is active in e11.5 whisker pads. (B) Lef1 is active in the whole e11.5 whisker 476 pad, coherently with the homogeneous expression of the first dermal signal. (C) Multispecies 477 alignment showing a primate specific deletion of a sequence adjacent to a conserved region. 478 Species analyzed include animals with developed whiskers (i.e. rat, cat, dog, rabbit, guinea pig, 479 squirrel, horse, naked mole rat, pig), primates (human, chimp, gorilla, gibbon, rhesus, baboon, 480 squirrel money, orangutan, marmoset, tarsier) and the human lineages. (D) HiC profile around Lef1 481 in murine ES cells (Dixon et al. 2012). Cen: centromeric. Tel: telomeric

\section{Figure 7}

(A) 4C-Seq on e12.5 whisker pad and adult kidney (negative control). In green, the Lef1 promoter (viewpoint, black arrow) contacts Leaf more frequently in the e12.5 whisker pad compared to the adult kidney. In blue, Leaf (viewpoint, black arrow) has a higher number of contacts on Lef1 promoter and coding sequence in e12.5 embryos compared to the adult kidney. Tracks are normalized (for profile-corrected tracks see Supplementary Figure 6). (B) Normalized, profile corrected scores of Lef1 promoter viewpoint on Leaf and vice versa show a statistically significant contact between the 12.5 whisker pad and adult kidney. (C) AR IHC shows no expression in the E12.5 whisker pad compared to dorsal root ganglia (positive control).

\section{Supplementary Figure 1}

494 Schematic representation of transgenic mouse lines used for this study.

\section{Supplementary Figure 2}

497 (A) Prdm1 IHC on developing head/back pelage hair follicles. Prdm1 expression in pelage hair follicle development is transient, lasts until hair germ formation (S2) and is independent of the embryonic origin of the mesenchyme. Dashed circles envelop the areas where Prdm1 is expressed. Scale bar: $50 \mu \mathrm{m}$. (B) WT e12.5 embryo compared to Prdm1 cKO1. cKO1 embryos lack 2/3 digits and display no whisker placodes macroscopically.

\section{Supplementary Figure 3}

504 (A) Expression of Sox2 in wild type and Prdm1 cKO1 whisker pad at e13.5. No dermal condensate 505 can be detected in cKO1 embryos. Scale bar: $500 \mu \mathrm{m}$ (B) Edar and Krt17 real time qPCR on E13 506 whisker pads of wild type and cKO1 mice.

\section{Supplementary Figure 4}

509 Prdm1Cre lineage tracing in developing whisker pads. Prdm1Cre was crossed with a ROSAYFP 510 strain and developing whisker pads were analyzed at e12.5 and e13. YFP positive cells encompass 511 the developing whisker. Scale bar: $50 \mu \mathrm{m}$.

512

\section{Supplementary Figure 5}

514 Wnt1Cre driven homozygous Prdm1 knockout mice (cKO2) lack almost all the macro vibrissae 515 except for the 1-3 distal ones of the first row, as observed both macroscopically and 
516 microscopically $(C, D, E)$ compared to wild type animals $(A, B)$. The barrel cortexes of the 517 correspondent animals were stained with cytochrome oxidase and reveal a major rearrangement.

518 Residual macro vibrissae are represented by enlarged barrels; the barrels representing micro 519 vibrissae are still present, though highly disorganized.

520

\section{Supplementary Figure 6}

522 Profile-corrected tracks of 4C-Seq on e12.5 whisker pad and adult kidney (negative control). In 523 green, the Lef1 promoter (viewpoint) contacts Leaf more frequently in the e12.5 whisker pad 524 compared to the adult kidney. In blue, Leaf (viewpoint) has a higher number of contacts on Lef1 525 promoter and coding sequence in e12.5 embryos compared to the adult kidney.

526 
Supplementary table 1 - List of genotyping primers

528

\begin{tabular}{|c|c|}
\hline \multirow[t]{2}{*}{ Generic Cre } & Primer Fw: CTAGAGCCTGTTTTGCACGTTC \\
\hline & Primer Rv: GTTCGCAAGAACCTGATGGACA \\
\hline \multirow[t]{2}{*}{ Prdm1 Cre } & Primer Fw: GCCGAGGTGCGCGTCAGTAC \\
\hline & Primer Rv: CTGAACATGTCCATCAGGTTCTTG \\
\hline \multirow[t]{3}{*}{ Lef1tmRug } & Primer 24: CCGTTTCAGTGGCACGCCCTCTCC \\
\hline & Primer 25: TGTCTCTCTTTCCGTGCTAGTTC \\
\hline & Primer 26: ATGGCGATGCCTGCTTGCCGAATA \\
\hline \multirow[t]{4}{*}{ ROSAYFP } & Primer: AAGGGAGCTGCAGTGGAGTA \\
\hline & Primer: CCGAAAATCTGTGGGAAGTC \\
\hline & Primer: ACATGGTCCTGCTGGAGTTC \\
\hline & Primer: GGCATTAAAGCAGCGTATCC \\
\hline \multirow[t]{4}{*}{ Prdm1loxlox } & Primer common A: \\
\hline & CCTGGTTAGTAGTTGAATGGGAGC \\
\hline & Primer F1A: GTGCTTTTCTTGTGTTGGGAGG \\
\hline & Primer F2A: AGCAGTGTTTCTGAGTGGGTGG \\
\hline
\end{tabular}

529

Supplementary table 2 - List of antibodies and dilutions

533

\begin{tabular}{|l|l|l|l|l|}
\hline Antibody & Species & Dilution & Clone & Company \\
\hline Prdm1 & Mouse & $1: 500$ & $3 \mathrm{H} 2-\mathrm{E} 8$ & Abcam \\
\hline Sox2 & Mouse & $1: 2000$ & $9-9-3$ & Abcam \\
\hline p75 & Mouse & $1: 1000$ & 9 G395 & UsBiologicals \\
\hline GFP & Goat & $1: 1000$ & 6673 & Abcam \\
\hline CD31 & Rat & $1: 500$ & MEC13.3 & BD Biopharmingen \\
\hline Ng2 & Rabbit & $1: 300$ & AB5320 & Millipore \\
\hline AR & Rabbit & $1: 5000$ & PG-21 & Millipore \\
\hline Tuj1 & Goat & $1: 300$ & MMS-435P & Covance \\
\hline Tuj1 & Rabbit & $1: 300$ & MRB-435P & Covance \\
\hline Pdgr $\beta$ & Rabbit & $1: 300$ & 28E1 & Cell Signaling \\
\hline GFP & Goat & $1: 1000$ & NB100-NB1770 & Novus \\
\hline
\end{tabular}

534 


\section{Supplementary table 3 - List of 4 C-Seq primers}

542

\begin{tabular}{|c|c|c|}
\hline Viewpoint & Name & Sequence \\
\hline \multirow[t]{3}{*}{ Lef1 promoter } & PromNla3ıllumina & 5'- AATGATACGGCGACCACCGAACACTCTTTCCCTACACG \\
\hline & & ACGCTCTTCCGATCTTTAAACAGGGCTACCCTTAAAACCA-3' \\
\hline & PromDpn5Illumina & $\begin{array}{l}\text { 5'- CAAGCAGAAGACGGCATACGAAGGCTCAGTCTTCATCC } \\
\text { ACACC-3' }\end{array}$ \\
\hline \multirow[t]{2}{*}{ Leaf } & EnNla2Illumina & $\begin{array}{l}\text { 5'- AATGATACGGCGACCACCGAACACTCTTTCCCTACACG } \\
\text { ACGCTCTTCCGATCTCCGGAAGCGGCTGTTCTC-3' }\end{array}$ \\
\hline & EnDpn1Illumina & $\begin{array}{l}\text { 5'- CAAGCAGAAGACGGCATACGAGGTGGAGAACGGAACC } \\
\text { CAAG-3' }\end{array}$ \\
\hline \multirow[t]{2}{*}{ HoxD13 } & Nla Dpn Hxd13 iF1 & $\begin{array}{l}\text { 5'- AATGATACGGCGACCACCGAACACTCTTTCCCTACACG } \\
\text { ACGCTCTTCCGATCTAAAATCCTAGACCTGGTCA-3' }\end{array}$ \\
\hline & Nla Dpn Hxd13 iR1 & $\begin{array}{l}\text { 5'-CAAGCAGAAGACGGCATACGAGGCCGATGGTGCTGTA } \\
\text { TAGG-3' }\end{array}$ \\
\hline
\end{tabular}

543 
544

545

546

547

548

549

550

551

552

553

554

555

556

557

558

559

560

561

562

563

564

565

566

567

568

569

570

571

572

573

574

575

576

577

578

579

580

581

582

583

584

585

586

\section{Materials and Methods}

\section{Mice}

OF1 and C57/B6J2 mice were obtained from Charles River Breeding Laboratories. Prdm1MEGFP transgenic mice were kindly provided by Mitinori Saitou; Lef1 tm1 Rug mice were provided by Rudolf Grosschedl and Werner Held. Sox2Cre, Sox2CreERT2, Prdm1Cre, Wnt1Cre, Prdm1 CA, ROSAYFP, mice were obtained from the Jackson Laboratory. The strains carrying the Cre recombinase and ROSAYFP were kept in heterozygosity, while the conditional knockout was performed in homozygosity. All animals were maintained in a 12?h light cycle providing food and water ad libitum. Mice were killed by intra-peritoneal (i.p.) injection of pentobarbital. Experiments were conducted in accordance with the EU Directive (86/609/EEC) for the care and use of laboratory animals and that of the Swiss Confederation. Mating of adult female and male mice was carried out overnight. Time-pregnant mice were killed by injection of pentobarbital and uteri with embryos were removed by dissection.

\section{Genotyping}

List of primers is available in supplementary Table 1.

\section{In situ hybridization}

RNA-FISH were performed using 8- $\mu$ m paraffin sections. Digoxigenin-labeled probes for specific transcripts were prepared by PCR with primers designed using published sequences. The mRNA expression patterns were visualized by immunoreactivity with anti-digoxigenin horseradish peroxidase-conjugated Fab-fragments (Roche, Basel, Switzerland), according to the manufacturer's instructions. The amplification was carried out using the TSA Plus Cyanine 3/5 System (Perkin Elmer). The probe SP72-Bmp4 and Bmp2 were provided by Severine Urfer, Shh by the Duboule group, Lef1 by Anne Grapin-Botton. Gli1 and Wnt10b ISH were performed with the ACDBioscope.

\section{In vivo lineage tracing}

For lineage tracing experiment, Sox2CreERT2/RosaYFP pregnant females were induced at embryonic day e12 and e12.5 with $2 \mathrm{mg}$ tamoxifen and $1 \mathrm{mg}$ of progesterone (Sigma-Aldrich) by intraperitoneal injection. The transgenic animals were retrieved at e17 and perinatally and then processed for histology and immunostaining.

\section{Proliferation experiments}

For EdU experiment, pregnant female mice carrying Prdm1MEGFP embryos were injected with 200? $\mathrm{\mu l}$ of EdU (30? $\mathrm{mg} /$ ? $\mathrm{ml}$ ) and analyzed 2? $\mathrm{h}$ after the first injection. Embryos were retrieved, genotyped under the UV lamp and processed for histology and immunostaining.

\section{Histology and immunostaining}

All samples were removed and fixed overnight in $4 \%$ paraformaldehyde at 4$]^{\circ} \mathrm{C}$. Tissues were washed three times in PBS for 5 ? ? min and incubated overnight in $30 \%$ sucrose in PBS at 4$]^{\circ} \mathrm{C}$; eventually they were then embedded in OCT and kept at -80 ?] ${ }^{\circ} \mathrm{C}$. Sections of 10 ? $\mu \mathrm{m}$ thickness were cut using a CM3050S Leica cryostat (Leica Mycrosystems). 
587 Sections were incubated in blocking buffer (1\% BSA, 0.3\% Triton in PBS) for 1?h at room 588 temperature. Primary antibodies were incubated overnight at 4 ? $^{\circ}$. Sections were rinsed three 589 times in PBS and incubated with appropriate secondary antibodies diluted to 1:1000 and DAPI in 590 blocking buffer for 1 ? ?h at room temperature. Sections were again washed three times with PBS. 591 The primary antibodies used are listed in supplementary table 2. The following secondary 592 antibodies were used: anti-mouse, anti-rabbit, anti-rat, anti-goat, conjugated to Alexa Fluor 488, 593568 and 647 (Molecular Probes). Nuclei were stained in DAPI solution (1:2000) and slides were 594 mounted in DAKO fluorescent mounting medium. As for the AP reaction, SIGMAFAST Fast Red TR 595 was used and visualized by confocal microscopy (Leica) at $568 \mathrm{~nm}$.

596

Imaging

598 Fluorescence microscopy images were captured under the LSM 780 confocal microscope (Carl 599 Zeiss); transmission microscopy images were acquired with either the Olympus Ax70 or the Zeiss 600 Axioscope 2 Plus.

601

602 RT-PCR

603 Total RNA was isolated from the embryonic whisker pad using RNeasy Mini Kit (Qiagen) according 604 to the manufacturer's instructions. Total RNA was extracted, and $250 \mathrm{ng}$ of each sample were 605 reverse transcribed using the Superscript III enzyme and random primers (Life Technologies).

606

\section{Quantitative PCR}

608 For qPCR, 1? $\mu$ l of cDNA was amplified with the Taqman Universal Mastermix II (Life Technologies), 609 in a 10] $\mu$ l total reaction volume; $5 \mu \mathrm{l}$ of the Mastermix, $1,5 \mu \mathrm{l}$ of CDNA and 3,5 $\mu \mathrm{l}$ of assay mix were 610 included in the reaction. The primers were bought from Applied Biosciences or synthetized at IDT. 611 The Taqman assays were performed using a 79000 HT Fast Real Time PCR system (AB). For data 612 analysis, the mouse Eef1alpha, $\beta$-actin, Tbp and Gapdh housekeeping genes were used as internal 613 control. Gene expression profiling was achieved using the Comparative CT method (DDC ) of 614 relative quantification (Livak and Schmittgen, 2001) using the SDS 2.4 software (Applied 615 Biosystems).

616

617 Interspecies Sequence Comparison

618 The comparison of the conserved noncoding elements and deletions in mouse-rat, mouse-guinea 619 pig, mouse-squirrel, mouse-rabbit, mouse-human, mouse-chimp, mouse-gorilla, mouse620 orangutan, mouse-rhesus, mouse-dolphin, mouse-cow, mouse-cat, mouse-dog, mouse-horse, 621 mouse-elephant was done using the Vertebrate Multiz Alignment and Conservation Track in the 622 UCSC genome browser, using a window size of $2 \mathrm{~kb}$.

623

625 For each sample, we harvested at least $1 \times 107$ cells and obtained 7-10 $\mu \mathrm{g}$ of output double626 digested, double-ligated DNA. We collected 120 embryonic whisker pads both at e12.5 and e13. 627 Nlalll and Dpnll were used as primary and secondary cutter respectively; ligation was performed 628 by using the Concentrated T4 DNA ligase from Promega. Primer sets for Lef1 promoter and 629 enhancer are described in Supplementary table 2. 
630 The primer set for HoxD13 has already been published. PCRs were multiplexed and sequenced 631 with Illumina's HiSeq2000. Raw data were subject to demultiplexing, mapping (mm10) and 4C 632 analysis through the HTS station (http://htsstation.epfl.ch) according to previously described 633 procedures. All figures were made using a running mean algorithm with a window size of eleven 634 fragments. The regions excluded for analysis are for the promoter track chr3: 131104979635131112546 and for the enhancer tracks chr3: 131016310-131022769. Normalization was done by 636 dividing the fragment scores by the mean of fragments scores falling into a region defined as +/$6371 \mathrm{Mb}$ (parameter) around the center of the bait coordinates.

\section{Statistical analysis}

640 The normalized and profile corrected values were processed with Prism. For Leaf, fragments of 641 region Chr3:131008663-131026430 (mm10) were analyzed whereas for the Lef1 promoter and 642 coding sequence, the fragments of region Chr3:131106987-131227057 were analyzed. Normality 643 of the data was excluded by D'Agostino \& Pearson omnibus normality test. Statistical differences 644 were assessed by applying an unpaired non-parametric two-tailed Mann-Whitney test. Differences 645 were regarded as significant if $p<0.05$, and significances are shown in figures as $*(* p<0.05, * * p$ $646<0.01, * * * p<0.001, * * * * p \leq 0.001)$. Analyses were conducted using GraphPad Prism version 8.0.

\section{Acknowledgements}

649 We thank Mitinori Saitou and Azim Surani for providing the Prdm1mEGFP mice, Rudolf Grosscheldl 650 and Werner Held for providing the Lef1tm1Rug mice, Irene Pizzitola for breeding and mating 651 Lef1tm1Rug mice, Michiko Kanemitsu for experimental support and constructive discussion, Giulio 652 Cossu for the suggestions on the characterization of the pericytes and proofreading the 653 manuscript, the EPFL CPG (Emilie Gesina, Gisele Ferrand) and the animal house of Epalinges 654 (Francis Derouet, Lisa Arlandi) for mice handling, Orbicia Riccio, Elisabeth Joye and Severine Urfer 655 for sharing protocols and probes for in situ hybridization, Andrea Zaffalon and Matteo Pluchinotta 656 for molecular biology suggestions, Matteo Pluchinotta for artwork, Olga de Sousa Silva and Lai 657 Quiewen for excellent technical help.

658

\section{Author contribution}

660 P.G.M. conceptualization, experimental work, manuscript writing; F.D. conceptualization and 661 experimental work on evolutionary part of the paper, manuscript writing; M.L. bioinformatic 662 analysis; J.C., B.M., F.D, M.S. experimental work; G.F.M and H.A. histology, immunohistochemistry 663 and ACDbioscope RNA-FISH; Y.B. conceptualization and supervision of the study.

\section{Competing financial interests}

666 The authors declare no competing financial interests.

\section{Financial support}

669 YB was supported by SNF grants 135578 and 156812. 


\section{Correspondence}

674 Correspondence to: pierluigi.manti@unimi.it; yann.barrandon@epfl.ch

\section{Bibliography}

Andrés, F.L. and Van der Loos, H. 1985. From sensory periphery to cortex: the architecture of the barrel field as modified by various early manipulations of the mouse whiskerpad. Anatomy and embryology 172(1), pp. 11-20. plasma cell fate is associated with Blimp-1 expression in vivo. Journal of Immunology 165(10), pp.

5462-5471.

Attanasio, C., Nord, A.S., Zhu, Y., et al. 2013. Fine tuning of craniofacial morphology by distantacting enhancers. Science 342(6157), p. 1241006.

686 Brecht, M. 2007. Barrel cortex and whisker-mediated behaviors. Current Opinion in Neurobiology 687 17(4), pp. 408-416.

688 Chen, C.-K., Symmons, O., Uslu, V.V., et al. 2013. TRACER: a resource to study the regulatory architecture of the mouse genome. BMC Genomics 14, p. 215.

690 Darbellay F, Necsulea A. Comparative Transcriptomics Analyses across Species, Organs, and 691 Developmental Stages Reveal Functionally Constrained IncRNAs. Mol Biol Evol. 2020 Jan 692 1;37(1):240-259.

693 De Gendt, K., Swinnen, J.V., Saunders, P.T.K., et al. 2004. A Sertoli cell-selective knockout of the 694 androgen receptor causes spermatogenic arrest in meiosis. Proceedings of the National Academy 695 of Sciences of the United States of America 101(5), pp. 1327-1332.

696 Diamond, M.E., von Heimendahl, M., Knutsen, P.M., Kleinfeld, D. and Ahissar, E. 2008. "Where" 697 and "what" in the whisker sensorimotor system. Nature Reviews. Neuroscience 9(8), pp. 601-612.

698 Dixon, J.R., Selvaraj, S., Yue, F., Kim, A., Li, Y., Shen, Y., Hu, M., Liu, J.S., Ren, B. Topological domains in mammalian genomes identified by analysis of chromatin interactions. Nature 485, 376-380,

701 Erzurumlu, R.S. and Gaspar, P. 2012. Development and critical period plasticity of the barrel cortex. The European Journal of Neuroscience 35(10), pp. 1540-1553.

703 Hardy, M.H. 1992. The secret life of the hair follicle. Trends in Genetics 8(2), pp. 55-61. Horsley,

704 V., O'Carroll, D., Tooze, R., et al. 2006. Blimp1 defines a progenitor population that governs

705 cellular input to the sebaceous gland. Cell 126(3), pp. 597-609.

706 Hayashi, S., Lewis, P., Pevny, L. \& McMahon, A. P. Efficient gene modulation in mouse epiblast

707 using a Sox2Cre transgenic mouse strain. Gene Expr. Patterns 2, 93-97 (2002).

708 Jahoda, C.A. and Oliver, R.F. 1984. Histological studies of the effects of wounding vibrissa follicles

709 in the hooded rat. Journal of embryology and experimental morphology 83, pp. 95-108.

710 Keller, A.D. and Maniatis, T. 1991. Identification and characterization of a novel repressor of 711 betainterferon gene expression. Genes \& Development 5(5), pp. 868-879.

712 Kvon, E. Z., Kamneva, O. K., Melo, U. S., Barozzi, I., Osterwalder, M., Mannion, B. J., Tissières, V., 713 Pickle, C. S., Plajzer-Frick, I., Lee, E. A., Kato, M., Garvin, T. H., Akiyama, J. A., Afzal, V., Lopez-Rios,

714 J., Rubin, E. M., Dickel, D. E., Pennacchio, L. A., \& Visel, A. (2016). Progressive Loss of Function in a 715 Limb Enhancer during Snake Evolution. Cell, 167(3), 633-642.e11. 
716 Kratochwil, K., Dull, M., Farinas, I., Galceran, J. and Grosschedl, R. 1996. Lef1 expression is

717 activated by BMP-4 and regulates inductive tissue interactions in tooth and hair development.

718 Genes \& Development 10(11), pp. 1382-1394.

719 Liu, X., Driskell, R.R., Luo, M., et al. 2004. Characterization of Lef-1 promoter segments that

720 facilitate inductive developmental expression in skin. The Journal of Investigative Dermatology

721 123(2), pp. 264-274.

722 Magnúsdóttir, E., Kalachikov, S., Mizukoshi, K., et al. 2007. Epidermal terminal differentiation

723 depends on B lymphocyte-induced maturation protein-1. Proceedings of the National Academy of

724 Sciences of the United States of America 104(38), pp. 14988-14993.

725 Martins, G.A., Cimmino, L., Shapiro-Shelef, M., et al. 2006. Transcriptional repressor Blimp-1

726 regulates T cell homeostasis and function. Nature Immunology 7(5), pp. 457-465.

727 McGowan, K.M. and Coulombe, P.A. 1998. Onset of keratin 17 expression coincides with the

728 definition of major epithelial lineages during skin development. The Journal of Cell Biology 143(2),

729 pp. 469-486.

730 McLean, C.Y., Reno, P.L., Pollen, A.A., et al. 2011. Human-specific loss of regulatory DNA and the

731 evolution of human-specific traits. Nature 471(7337), pp. 216-219.

732 Millar, S. E. 2002. Molecular mechanisms regulating hair follicle development. Journal of

733 Investigative Dermatology 118, pp.216-225

734 Noramly, S., Freeman, A. and Morgan, B.A. 1999. beta-catenin signaling can initiate feather bud

735 development. Development 126(16), pp. 3509-3521.

736 Ohinata, Y., Payer, B., O'Carroll, D., et al. 2005. Blimp1 is a critical determinant of the germ cell

737 lineage in mice. Nature 436(7048), pp. 207-213.

738 Oliver, R.F. 1966. Whisker growth after removal of the dermal papilla and lengths of follicle in the 739 hooded rat. Journal of embryology and experimental morphology 15(3), pp. 331-347.

740 Petersen, C.C.H. 2007. The functional organization of the barrel cortex. Neuron 56(2), pp. 339-355.

741 Robertson, E.J., Charatsi, I., Joyner, C.J., et al. 2007. Blimp1 regulates development of the posterior

742 forelimb, caudal pharyngeal arches, heart and sensory vibrissae in mice. Development 134(24), pp.

743 4335-4345.

744 Shaffer, A.L., Lin, K.I., Kuo, T.C., et al. 2002. Blimp-1 orchestrates plasma cell differentiation by

745 extinguishing the mature B cell gene expression program. Immunity 17(1), pp. 51-62. Shapiro-

746 Shelef, M., Lin, K.-I., McHeyzer-Williams, L.J., Liao, J., McHeyzer-Williams, M.G. and Calame, K.

747 2003. Blimp-1 is required for the formation of immunoglobulin secreting plasma cells and pre-

748 plasma memory B cells. Immunity 19(4), pp. 607-620.

749 Shimizu, T., Morita, W. and Maeda, T. 2013. Genetic mapping of agenesis of the third molars in 750 mice. Biochemical Genetics 51(9-10), pp. 728-736.

751 Tamatsu, Y., Tsukahara, K., Hotta, M. and Shimada, K. 2007. Vestiges of vibrissal capsular muscles 752 exist in the human upper lip. Clinical Anatomy 20(6), pp. 628-631.

753 van Genderen, C., Okamura, R.M., Fariñas, I., et al. 1994. Development of several organs that 754 require inductive epithelial-mesenchymal interactions is impaired in LEF-1-deficient mice. Genes \& 755 Development 8(22), pp. 2691-2703.

756 Van Horn, R.N. 1970. Vibrissae structure in the rhesus monkey. Folia primatologica; international

757 journal of primatology 13(4), pp. 241-285. 
758 Vincent, S.D., Dunn, N.R., Sciammas, R., et al. 2005. The zinc finger transcriptional repressor 759 Blimp1/Prdm1 is dispensable for early axis formation but is required for specification of primordial 760 germ cells in the mouse. Development 132(6), pp. 1315-1325.

761 Woolsey, T.A. and Van der Loos, H. 1970. The structural organization of layer IV in the 762 somatosensory region (SI) of mouse cerebral cortex. The description of a cortical field composed 763 of discrete cytoarchitectonic units. Brain Research 17(2), pp. 205-242.

764 Yeh, S., Tsai, M.-Y., Xu, Q., et al. 2002. Generation and characterization of androgen receptor 765 knockout (ARKO) mice: an in vivo model for the study of androgen functions in selective tissues. 766 Proceedings of the National Academy of Sciences of the United States of America 99(21), pp. 767 13498-13503. 
A

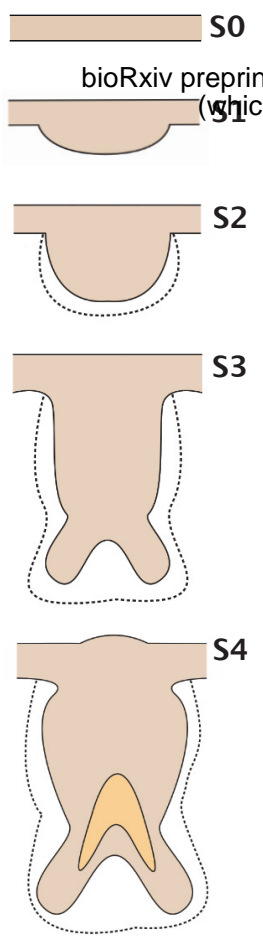

C

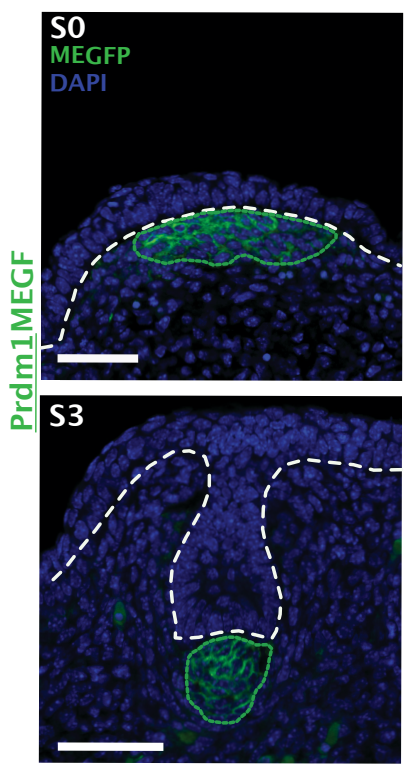

B
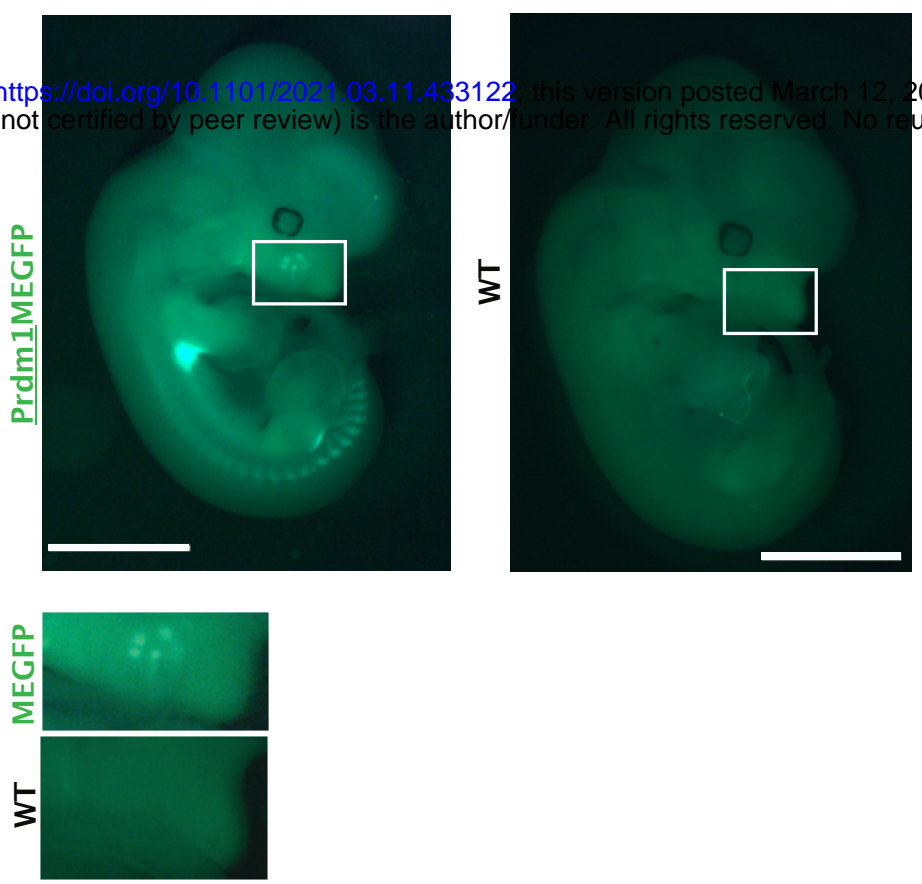

D

S1
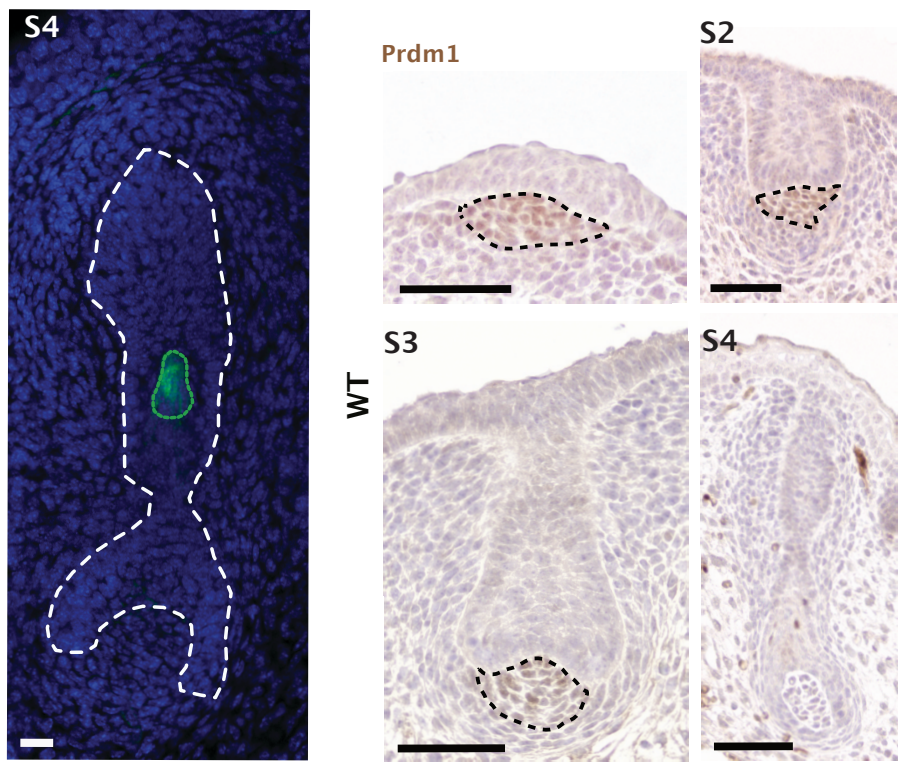

Figure 1

021. The copyright holder for this preprint use allowed without permission. 
Figure 2

A

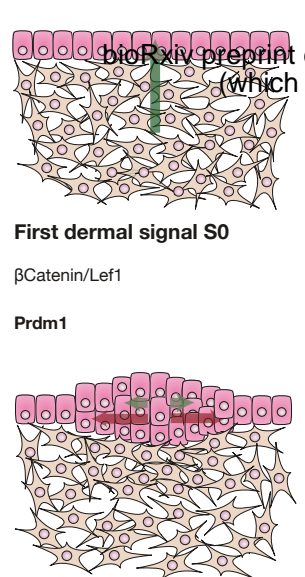

Promotion of placodal fate (green) S1

Wnt10b

$\beta$ Catenin/Lef1

Fgf10/Fgfr2-IIlb

Eda/Edar

Noggin?

Delta-1/Notch1?

Inhibition of placodal fate (red) Bmp2/Bmp4/Bmpr1a

Delta-1/Notch1?

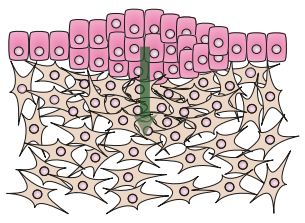

First epidermal signal S1

Wnt

Shh

Gli1
WT
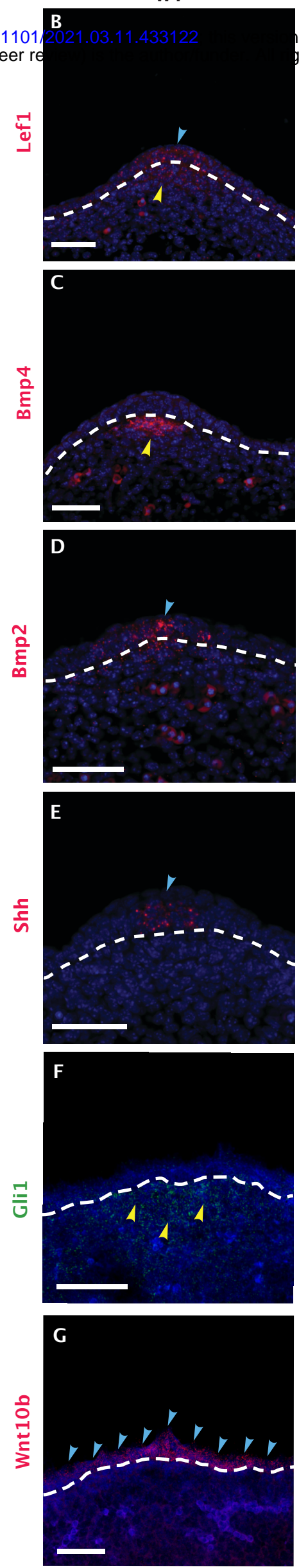

Prdm1 cKO1
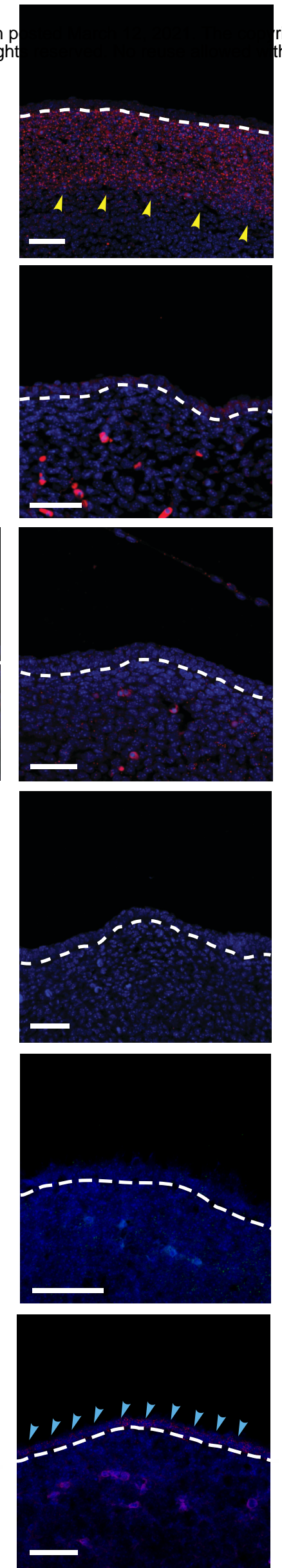

right holder for this preprint hout permission. 
Figure 3
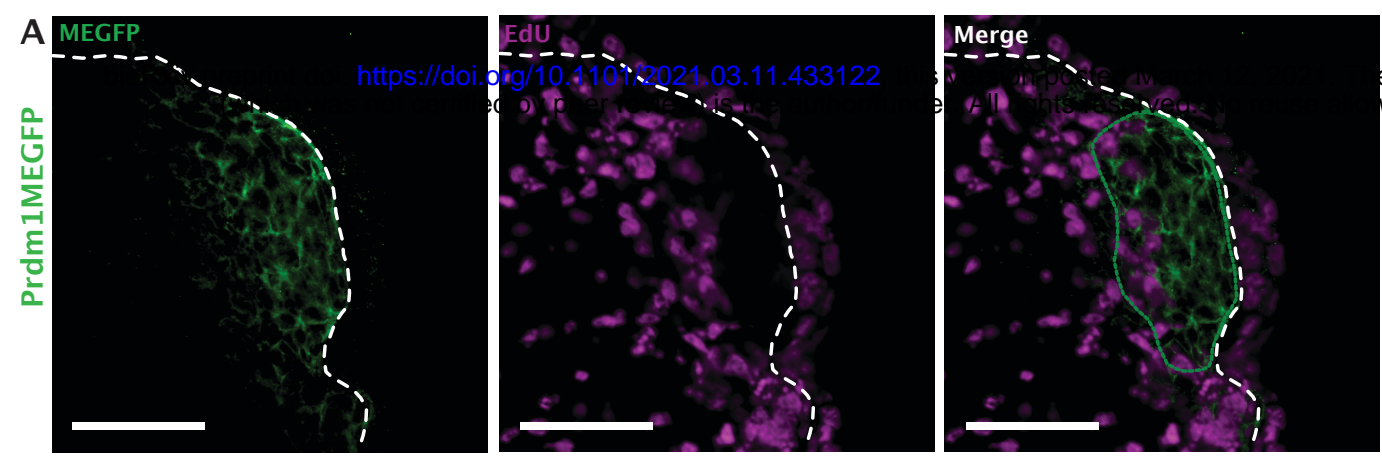

copyright holder for this preprint ved without permission.
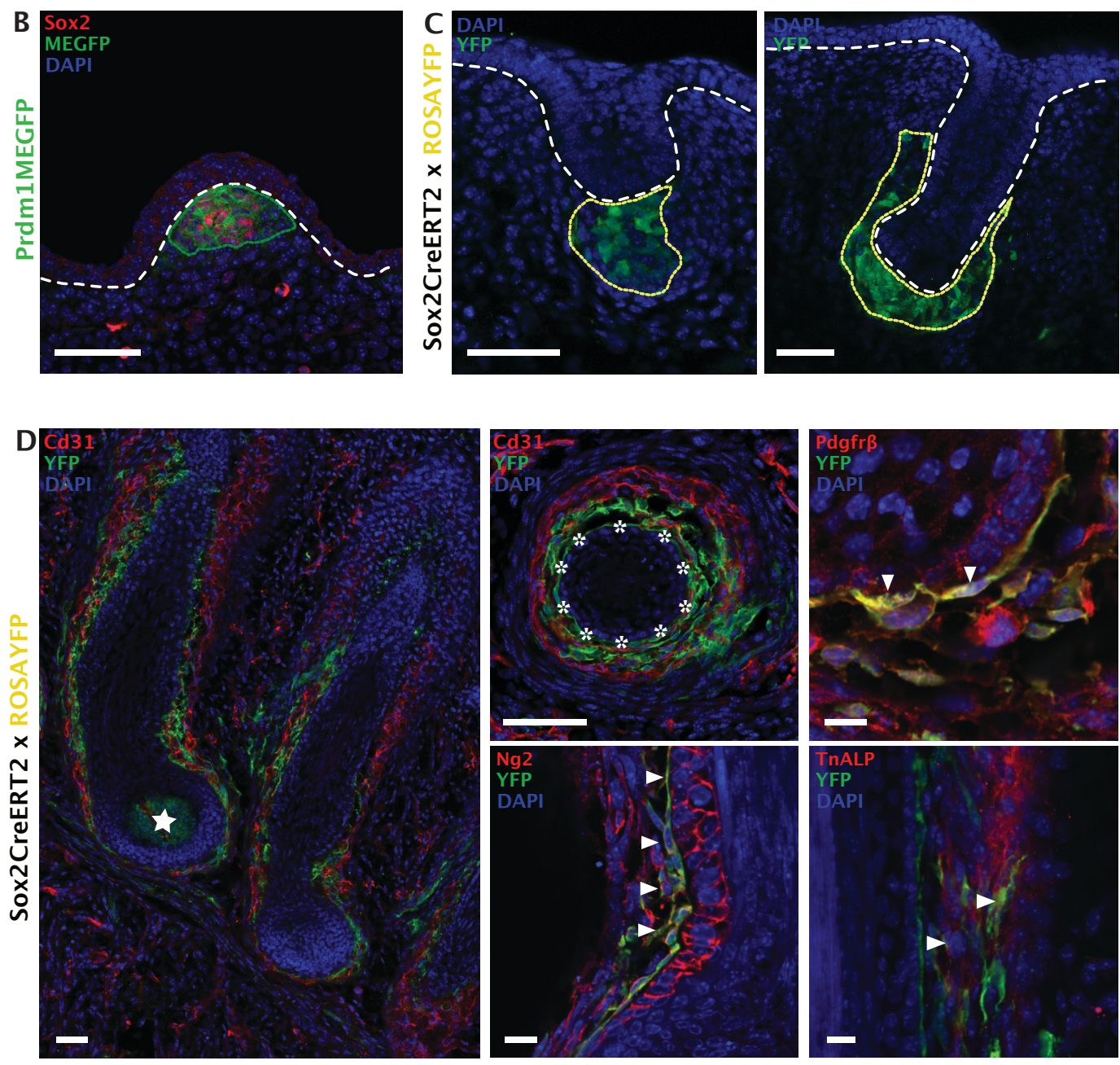
Figure 4
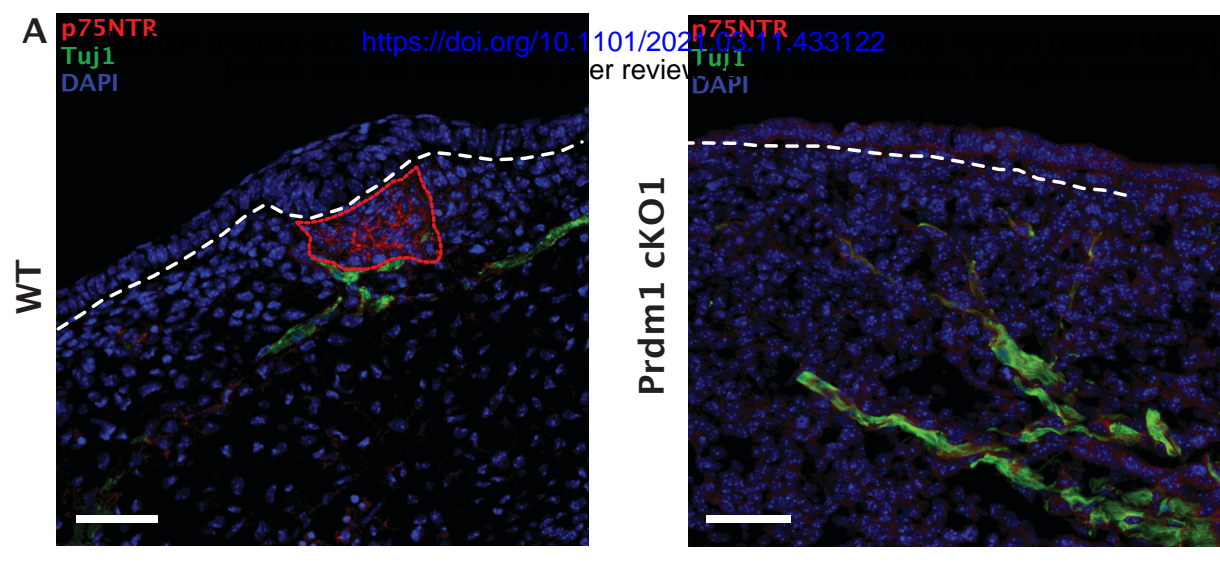

12, 2021. The copyright holder for this preprint lo reuse allowed without permission.

B

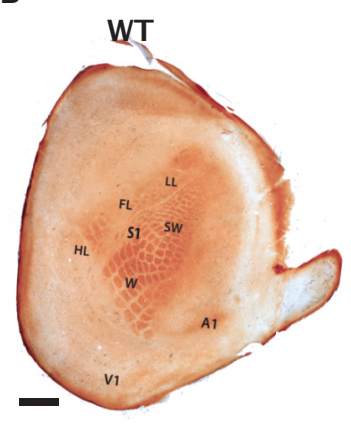

Prdm1 cKO2
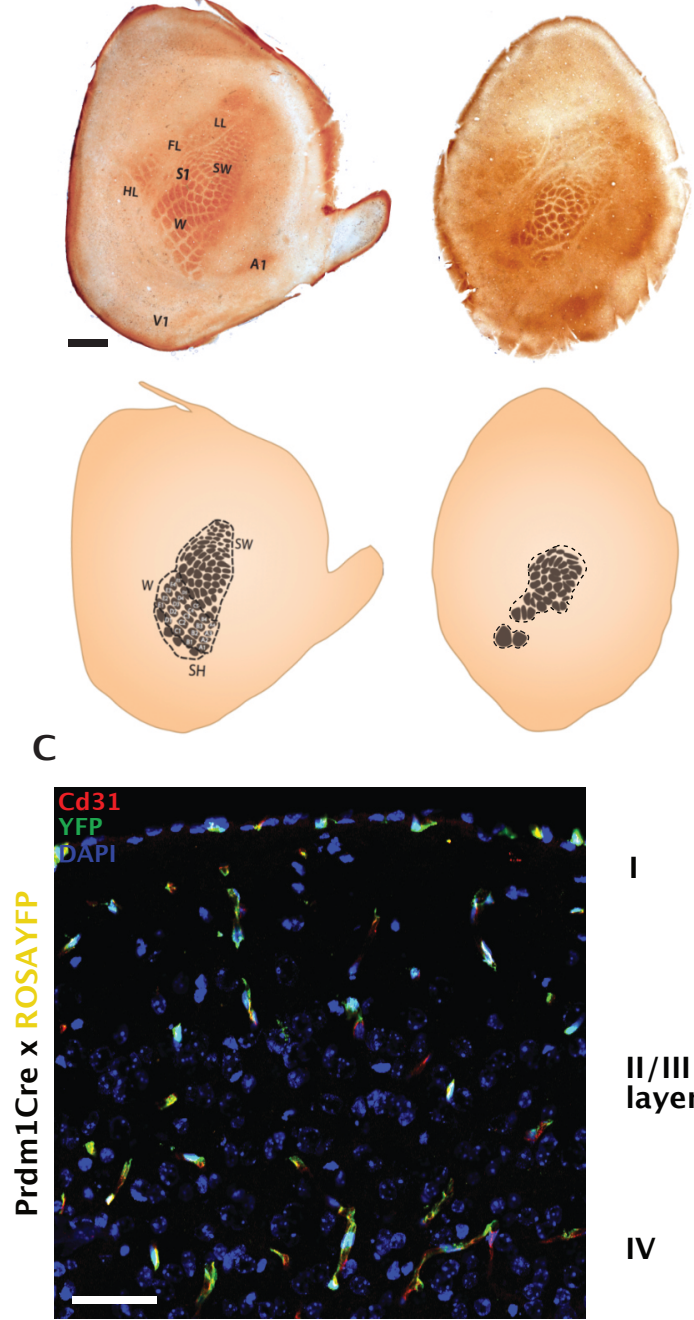

II/III (external granular/pyramidal

layer)

IV 
A

bioRxiv preprint doi: https://doi.org/10.1101/2021.03.11.433122; this version posted March 12, 2021. The copyright holder for this preprint

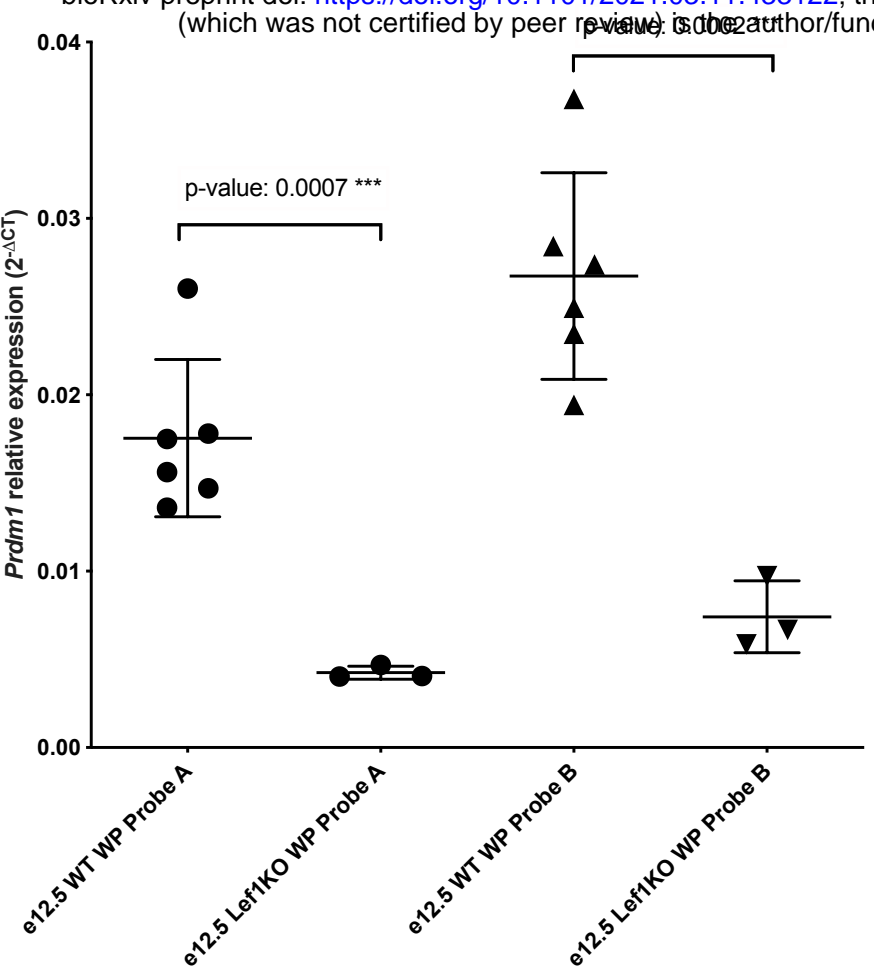

B

WT

Prdm1
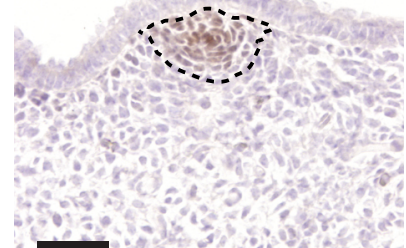

\section{Lef1 KO}

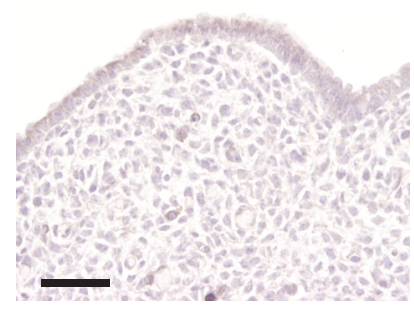

C

\begin{tabular}{|l|l|l|}
\hline Lef KO \# & $\begin{array}{l}\text { Prdm1 + } \\
\text { domes }\end{array}$ & $\begin{array}{l}\text { Prdm1 + } \\
\text { domes }\end{array}$ \\
\hline KO 6 & $2 / 28$ & $7 \%$ \\
\hline KO 8 & $4 / 27$ & $14 \%$ \\
\hline KO 4,5,7 & $0 / 28$ & $0 \%$ \\
\hline
\end{tabular}


bioRxiv preprint doi: https://doi.org/10.1101/2021.03.11.433122; this version posted March 12, 2021. The copyright holder for this preprint (which was not certified by peer review) is the author/funder. All rights reserved. No reuse allowed without permission.
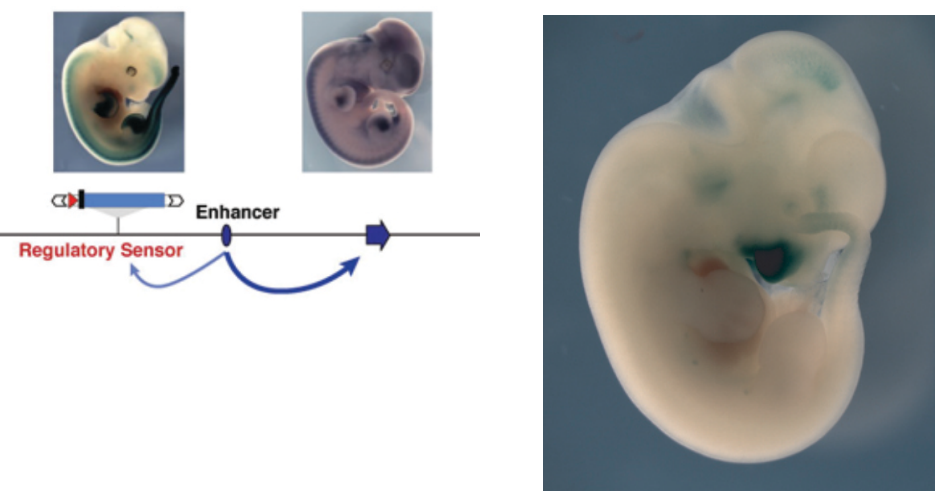

C

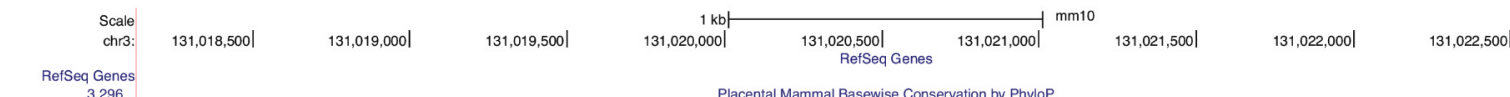

Placental Cons 3.296 _ Placental Mammal Basewise Conservation by PhyloP

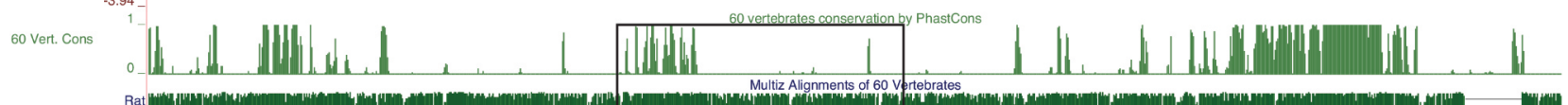

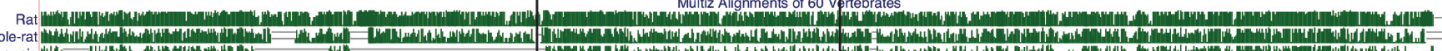

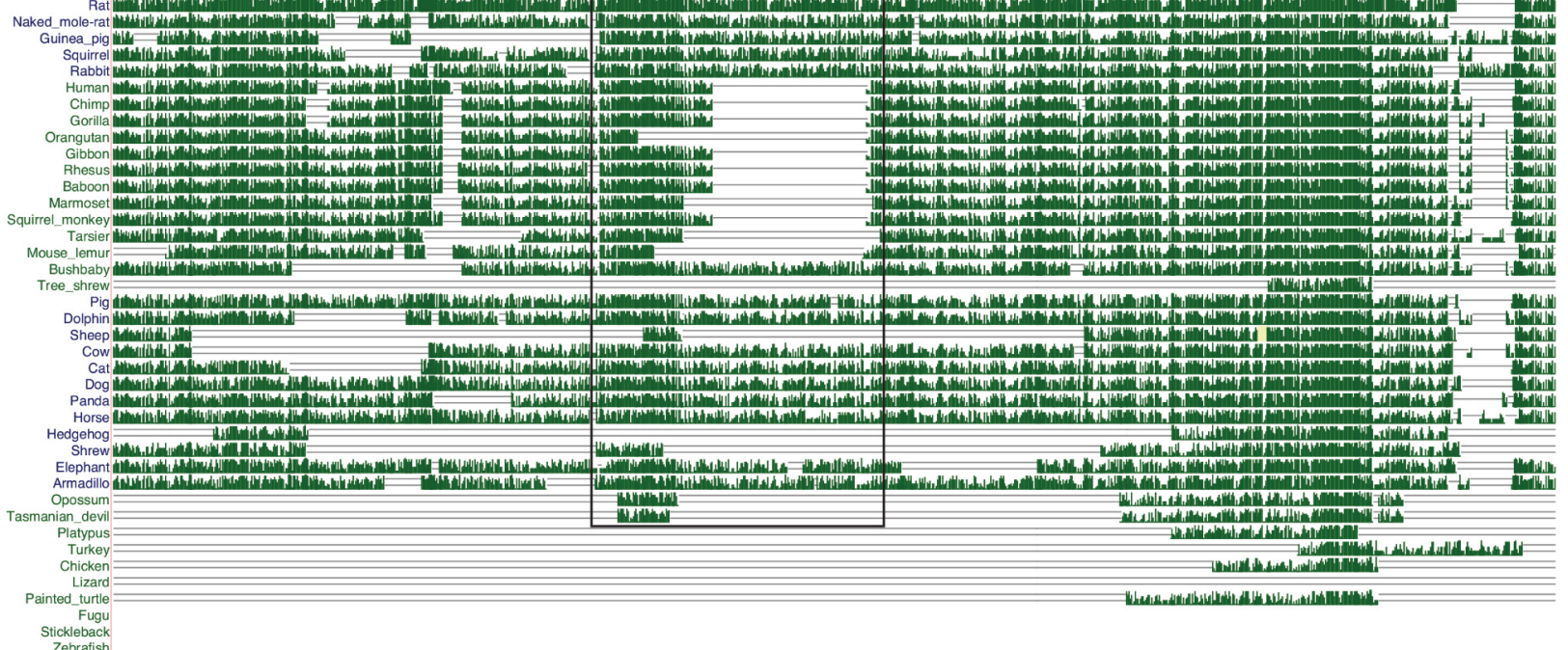

D

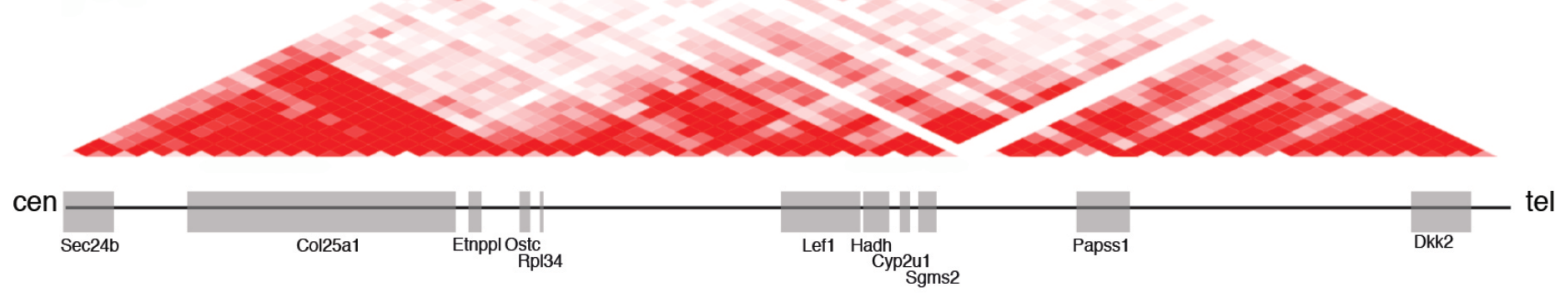


A bioRxiv preprint doi: https://doi.org/10.1101/2021.03.11.433122; this version posted March 12, 2021. The $200 \mathrm{~kb}$
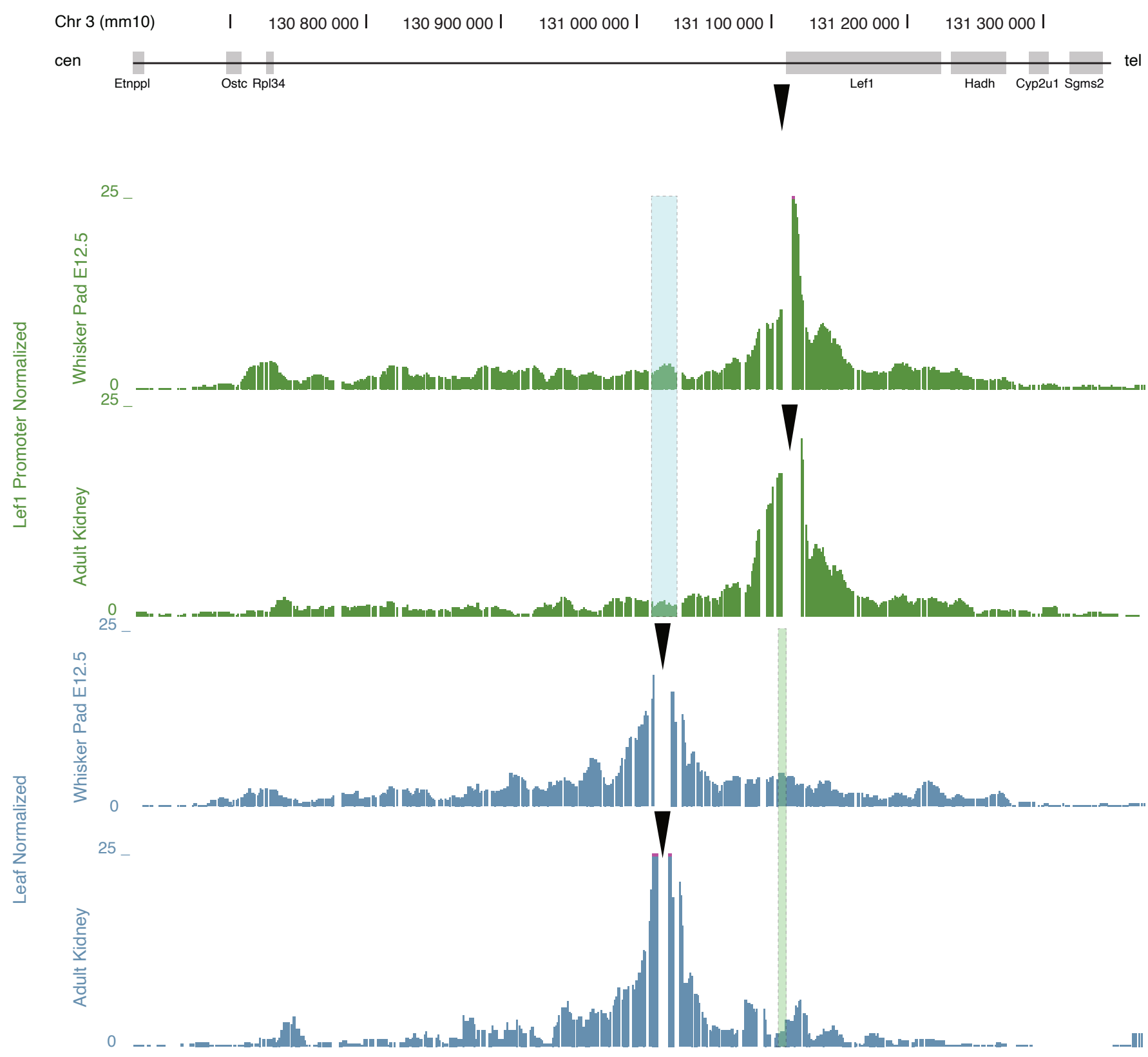

B

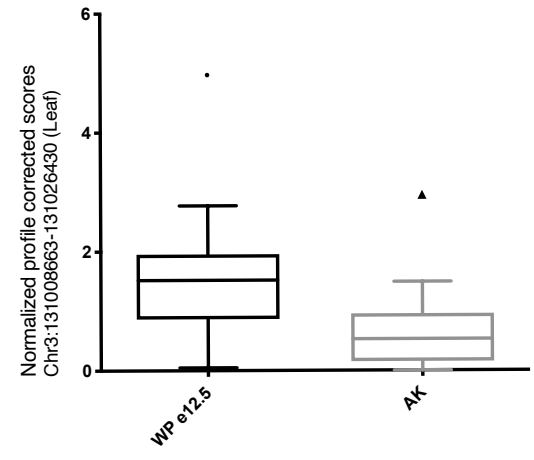

Lef1 promoter viewpoint

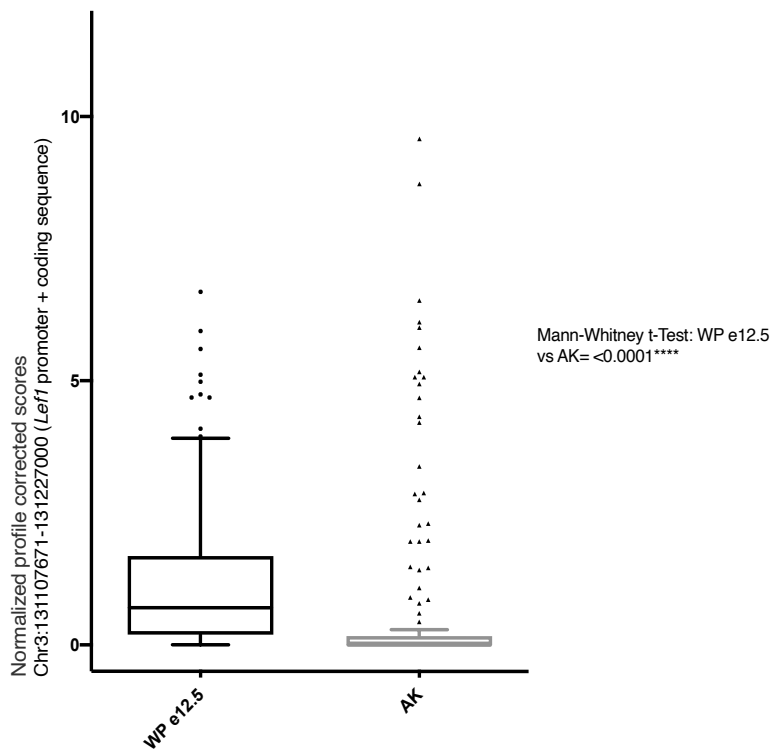

Leaf enhancer viewpoint 
